\title{
DISSECTING ANEURYSM OF THE CORONARY ARTERY IN ARACHNODACTYLY
}

\author{
BY \\ FLORENCE MCKEOWN \\ From the Department of Pathology, Queen's University of Belfast
}

A variety of cardiovascular lesions has been described in association with Marfan's syndrome, and these account for the sudden and premature death that occurs not infrequently in patients suffering from this disease. The following case is recorded as an example of arachnodactyly complicated by medial necrosis of the coronary vessels, leading to a dissecting aneurysm, with death from acute myocardial infarction.

\section{Case Report}

The patient was a young man, aged 21. Before his present illness his health had been good, except for a minor abdominal injury sustained 9 years previously. Three days before admission to hospital, he developed a pain in the left side of his chest which radiated down his left arm. The pain was sharp and stabbing and persisted for two days, after which it eased off for some hours. He then became more acutely ill, and when admitted complained of severe abdominal pain that recurred intermittently and was accompanied by vomiting.

On examination he was very pale and shocked, and lips, hands, and feet were cyanosed. Arachnodactyly was noted, but there were no other stigmata of Marfan's syndrome. The radial pulse was not palpable and the blood pressure was unrecordable. The venous pressure was raised and the cervical veins pulsating. The apex beat was visible in the fifth left interspace three inches from the sternum, and the heart rate was 120 a minute. A harsh systolic murmur was heard over the præcordium. An electrocardiogram showed the pattern of a large posterior myocardial infarct. The patient's condition deteriorated rapidly and he died four hours after admission.

\section{Necropsy}

The main pathological findings were confined to the heart, which weighed $300 \mathrm{~g}$. Externally there was subepicardial hæmorrhage along the posterior atrioventricular groove. The right atrium was moderately dilated and showed the presence of a transverse tear in the endocardium, $2 \mathrm{~cm}$. in length, situated just above the medial cusp of the tricuspid valve and below the opening of the coronary sinus. Necrotic muscle and blood clot extruded from the tear (Fig. 1). The right ventricle, pulmonary arteries, left atrium, and mitral valve appeared normal. The left ventricle was dilated and the wall was rather atrophic. A ragged tear was found in the posterior wall of the left ventricle close to the mitral ring. The surrounding muscle was soft and friable, and though little altered in colour appeared to be the site of a recent infarct (Fig. 2). The rupture did not extend through the wall of the ventricle to the external surface but communicated with a track that traversed the upper part of the interventricular septum posteriorly, to the wall of the right atrium and terminated in the endocardial tear already noted in this cavity. Macroscopic examination of the coronary arteries revealed no atheroma. The right vessel was somewhat dilated $1 \mathrm{~cm}$. from its origin, and appeared to be occluded by a thrombus at this level and for $2 \mathrm{~cm}$. along its course.

Microscopic Examination. A section of the right coronary artery at the level of occlusion revealed a large intramural hæmatoma which was bounded on its outer aspect by a few fibres of medial muscle, the external elastic lamina, and adventitial fibrous tissue, and on its inner aspect by an almost intact media. The vessel showed slight intimal fibrosis and the lumen was virtually closed by the displacement inwards of the media due to dissection of the outer coats by blood (Fig. 3). This dissection extended along the vessel for 


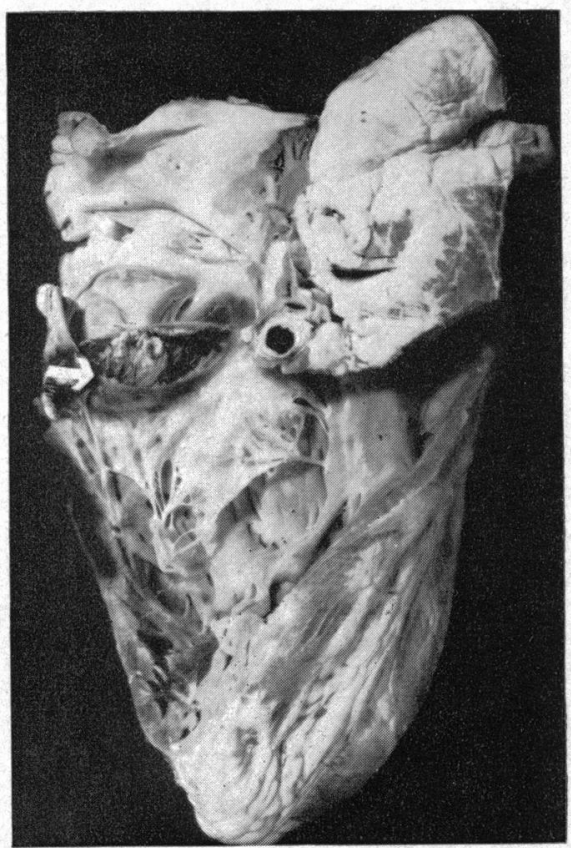

FIG. 1.-Arrow denotes transverse tear in right atrium. The occluded right coronary artery is also seen.

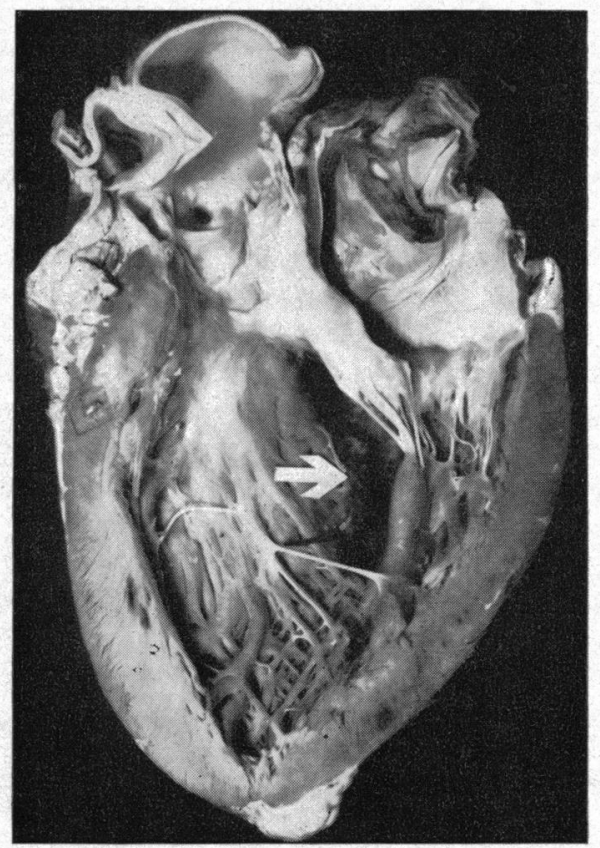

FIG. 2.-Left ventricle, with partially ruptured infarct in posterior wall.

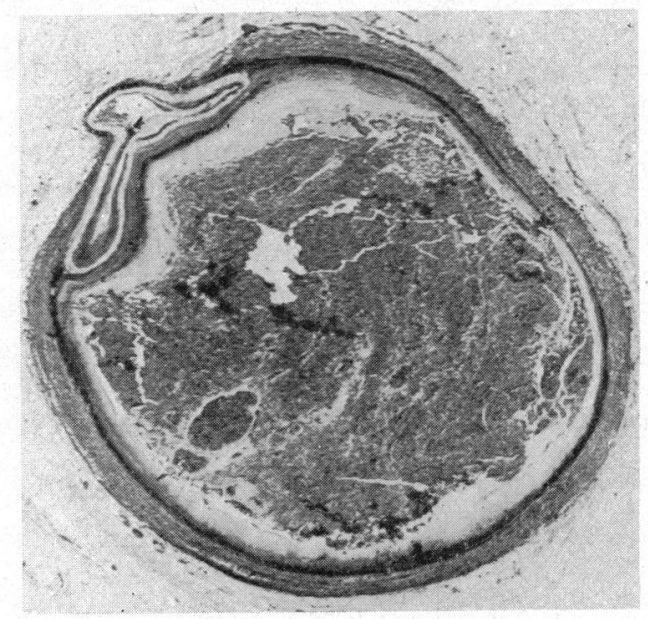

FIG. 3.-Right coronary artery, showing extent of dissection and compression of original lumen. (H.V.G. $\times 12$ ).

about $2 \mathrm{~cm}$. At its proximal limit the channel was narrow and surrounded by much fibrous tissue, but at its distal limit there was an abrupt breach in the medial muscle coat, which probably represented the point of emergence of the hæmatoma into the lumen. The surviving media in the vicinity of the dissection showed some degeneration with loss of muscle fibres and replacement fibrosis, but the extent of the dissection was so 
great that it masked most of the evidence of pre-existing medial necrosis. Blocks of the coronary vessels at a distance from the lesion showed a normal structure.

Histological examination of the left ventricle confirmed the presence of a recent infarct in the posterior wall.

\section{Comment}

Cardiovascular lesions have been reported in 30-60 per cent of cases of Marfan's disease. It has been suggested by Goyette and Palmer (1953) that they may occur as isolated lesions in some families with arachnodactyly and that all manifestations of the syndrome may not be present. The association of cardiovascular lesions and arachnodactyly in the present case may represent a "forme fruste" of the disease.

The most common cardiovascular manifestation is involvement of the aorta by a dissecting or fusiform aneurysm. Valvular thickening also occurs with some frequency. Histologically the aortic media shows a deficiency of elastic and muscle fibres, and the changes are indistinguishable from Erdheim's medial necrosis. They have been attributed variously to a congenital defect of the media, to a developmental anomaly of the nutrient vessels, or to a disturbance in ground substance due to some primary endocrine disease. Sometimes the aortic dissection has been associated with extension into the coronary and carotid vessels, but the first case of primary extraaortic dissection in this syndrome was recorded by Austin and Schaffer in 1957: the innominate and right common carotid vessels were involved in their case. The present case is the first described with dissection restricted to the coronary system. The changes of medial necrosis were largely obscured by the extent of the dissection, nevertheless it is assumed they were the basis for its development. Histological examination of the aorta and other vessels showed no similar degeneration.

Dissecting aneurysms have previously been described in the coronary arteries apart from Marfan's syndrome: the most recent report is by Boschetti and Levine (1958) who noted cystic medionecrosis of the vessels in four patients, in one of whom a dissecting aneurysm and myocardial infarction had developed. Aneurysms of the coronary arteries in any form are rare and Crocker et al. (1957) in a comprehensive review could trace only 67 previously reported examples.

The dissection in this case resulted in myocardial infarction in the territory of the affected vessel. Partial rupture of the infarct with dissection of blood through the wall of the heart to find an outlet in the cavity of the right atrium must be a most unusual sequence of events. This no doubt accounted for the sudden worsening of the patient's condition, the raised and pulsating jugular venous pressure, and the terminal circulatory failure.

\section{Summary}

A case of arachnodactyly associated with a dissecting aneurysm of the right coronary artery and myocardial infarction is presented. The infarct allowed dissection by blood to occur from the cavity of the left ventricle into the cavity of the right atrium.

Thanks are due to Dr. A. P. Grant of the Belfast City Hospital for access to the clinical notes of this patient. The photography is the work of Dr. D. Mehaffey, A.R.P.S.

\section{References}

Austin, M. G., and Schaffer, R. F. (1957). Arch. Path., 64, 205. Boschetti, A. E., and Levine, A. (1958). Arch. intern. Med., 102, 562. Crocker, D. W., Sobin, S., and Thomas, W. C. (1957). Amer.J. Path., 33, 819. Goyette, E. M., and Palmer, P. W. (1953). Circulation, 7, 373. 